\title{
A Free-Viewpoint Virtual Mirror with Marker-Less User Interaction
}

\author{
Matthias Straka, Stefan Hauswiesner, Matthias Rüther, and Horst Bischof \\ Institute for Computer Graphics and Vision, \\ Graz University of Technology, \\ Inffeldgasse 16/II, Graz, Austria \\ \{straka, hauswiesner, ruether, bischof\}@icg. tugraz.at
}

\begin{abstract}
We present a Virtual Mirror system which is able to simulate a physically correct full-body mirror on a monitor. In addition, users can freely rotate the mirror image which allows them to look at themselves from the side or from the back, for example. This is achieved through a multiple camera system and visual hull based rendering. A real-time $3 \mathrm{D}$ reconstruction and rendering pipeline enables us to create a virtual mirror image at 15 frames per second on a single computer. Moreover, it is possible to extract a three dimensional skeleton of the user which is the basis for marker-less interaction with the system.
\end{abstract}

Keywords: Virtual Mirror, Shape-from-Silhouette, CUDA, Markerless User-Interaction, Free-Viewpoint Video.

\section{Introduction}

A virtual mirror is an Augmented Reality (AR) system which renders an image of the user from a virtual viewpoint and creates the illusion of a mirror image. In addition to that, it allows to add virtual objects and to modify the image in a way that is not possible with physical mirrors. In this paper, we propose a physically correct simulation of a full-body mirror using a multi-camera system and a single monitor. We also extend its viewing capabilities by allowing users to rotate their mirror image using solely natural hand gestures. This is beneficial, for example, in dressing rooms where users want to see how they look from the side or from behind in new clothes.

The concept of a virtual mirror is not new in the area of computer vision and AR 568 . One common property of existing virtual mirror systems is that they consist of a single camera with a fixed position where the horizontally flipped video stream is shown to the user on a monitor. However, such systems generally do not allow one to simulate a correct mirror because video devices are only able to capture a projected image and not the reflection of the user from his viewpoint. Free viewpoint video systems on the other hand are able to render an arbitrary view of a person by combining images from multiple fixed cameras 3918 . This allows for the accurate simulation of optical effects, including rendering of a reflection image. Due to the amount of image data,

A. Heyden and F. Kahl (Eds.): SCIA 2011, LNCS 6688, pp. 635 645, 2011.

(C) Springer-Verlag Berlin Heidelberg 2011 
processing of the camera input often can not be performed in real-time on a single computer. Only recent developments in GPU computing have made it possible to render virtual viewpoints at interactive frame rates. We provide a more detailed review of the current literature in Sect. 2.

Our contribution is a novel virtual mirror system which combines a multicamera capture system with real-time free-viewpoint video. We equip a room with several cameras (see Sect. 3) and use a virtual camera to render an artificial mirror image which we display on a large monitor. Even though such a design incurs additional hardware compared to a single camera system, it adds several benefits. Due to the fact that the virtual camera has no restrictions on its position and parameters, we can generate an optically correct mirror effect. In Sect. 4 we describe how we determine and adapt the camera parameters when the user moves in front of the monitor. The capabilities of a multi-view camera system reach far beyond basic mirror simulations. We are able to not only show the frontal view of the user but rotate the virtual camera in a $360^{\circ}$ fashion around her. So far, our system is the only virtual mirror which provides such possibilities. The user can control this rotation by means of hand-gestures which we detect in a skeletal representation of the body. In Sect. 5 we show that we are able to achieve real-time processing of the camera images using only a single computer. We evaluate the robustness of user input detection and present a qualitative evaluation of the mirror image. Finally, Sect. 6 provides conclusions and a comparison to existing virtual mirror systems as well as suggestions for future enhancements of the proposed system.

\section{Related Work}

In this section we will give an overview of previous work concerning virtual mirrors and free-viewpoint video. Virtual mirrors have gained huge interest in recent years in the area of computer vision and augmented reality. For example, in 6] a camera is attached to a portable flat screen in order to simulate a hand-held mirror. Other virtual mirrors are able to display augmented objects or completely altered appearances instead of the true mirror image 2517. In fashion stores, people will use a mirror for looking at themselves in new clothes or with gadgets. This leads to the idea of using virtual mirrors for augmenting clothes [8] or shoes [4] onto the mirror image of a person without the need to actually wear them. Recently, commercial websites such as [1] have started to offer web cam applications which for example show an augmented mirror image of users with virtual sunglasses on their face. All these systems share the restriction of a single view-point: they only allow a frontal view of the user.

In contrast, free viewpoint video systems are able to render arbitrary views of an object by using images from multiple fixed cameras. There exist two major forms of object representation in such systems. Polygon based models [314] explicitly reconstruct the object as a triangle-mesh and use texture mapping to overlay the current video stream on the object. While such methods allow high quality outputs, they are only suitable for off-line processing due to the 


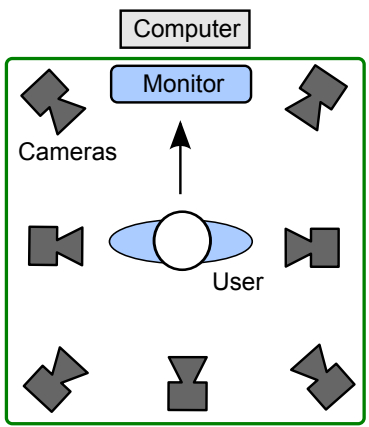

(a)

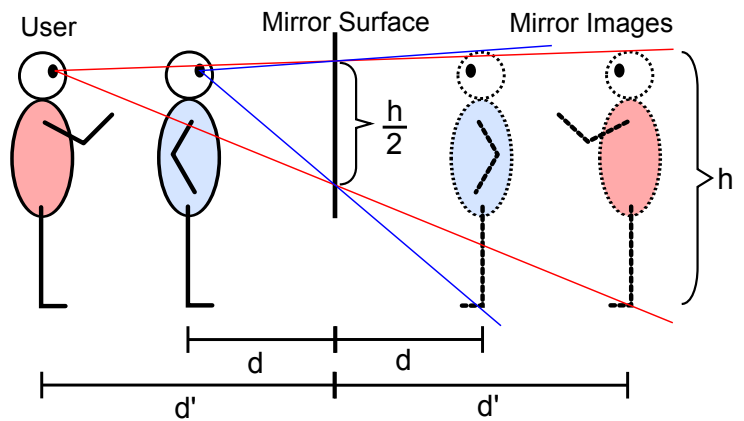

(b)

Fig. 1. (a) Top-view of the camera and virtual mirror setup: The user is surrounded by multiple cameras and sees an artificial mirror image of himself or herself on the display. (b) Illustration of the mirror effect: When the user has a distance $d$ from the mirror, the mirror image appears at the same distance behind the mirror. The height of the mirror image on the mirror surface always is half the height $h$ of the user and does not depend on the distance $d$.

computationally intensive reconstruction. Visual-hull based rendering [19] requires only depth information obtained through processing of camera images with background segmentation (silhouette) in order to synthesize a new viewpoint. However, true real-time processing of such data is still a challenging task. Only recent developments in GPU computing have made it possible to render virtual viewpoints at interactive frame rates [18, but often exceed the computational power of one computer [16].

\section{System Design}

Our virtual mirror system requires three components: multiple cameras to capture images of the user, a computer to process the data and a monitor to show the mirror image. Figure 1a sketches the setup and shows the positions of the user, the mirror display and the multi-view camera system.

\subsection{Geometric Considerations for the Mirror}

A virtual mirror needs to simulate the properties of a real mirror as accurate as possible. When standing in front of a mirror at a distance $d$, the mirror image of oneself appears exactly at the same distance but behind the mirror. However, even though this distance can change, the size of the user in the mirror image when projected on the mirror surface stays constant at half the height $h$ of the user. This is illustrated in Fig. 1b. We take advantage of this observation in order to choose an appropriate monitor for our mirror. A 47 inch Full-HD television mounted in portrait mode has a height of $104 \mathrm{~cm}$ and therefore can display the 


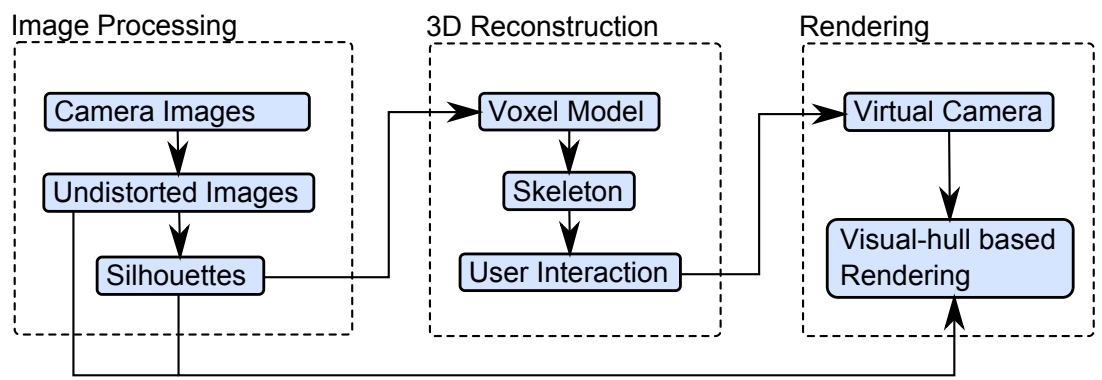

Fig. 2. Processing pipeline for the Virtual Mirror System

full-body mirror image of users up to 2 meters in height, which includes 99 percent of all people according to [15].

\subsection{System Architecture}

The complete setup is required to fit into a small room of $3 \times 2$ meters, which is equipped with ten synchronized color cameras connected to a single computer via three FireWire buses. Due to the space limitations, the viewing frustums of all cameras are focused on an area approximately 1.5 meters in front of the monitor, so the user is only allowed to move within a small area. The cameras deliver $640 \times 480$ pixel images at 15 frames per second. In order to process the amount of input data produced by our setup (around $100 \mathrm{MB} / \mathrm{s}$ ), we exploit the computational power of a CUDA enabled NVIDIA GTX 480 graphics card. Developing algorithms that execute on the GPU enables high performance in applications with many parallel tasks [10] such as pixel-wise image processing (e.g. image undistortion and silhouette extraction). Due to these hardware components, we are able to process the camera images and display an output on the virtual mirror at 15 frames per second using only a single computer.

\section{Methodology}

Our Virtual Mirror system uses a series of processing steps which extract the required information from the input images. We illustrate the pipeline that performs our reconstruction and rendering process in Fig. 2. In the first stage, we acquire a live stream of multiple synchronized camera images. Each image is then undistorted using radial distortion parameters determined during camera calibration. A subsequent silhouette extraction step segments the user from the background in all images. We employ a luminance insensitive background subtraction method in order to cope with shadows caused by the user. Silhouette data is used to generate a low-resolution voxel model and extract a skeleton which we use to implement marker-less user interaction. In the mirror simulation mode of our system, a virtual camera is controlled by the $3 \mathrm{D}$ position of the user's head which we determine in voxel space. An alternative mode of operation 


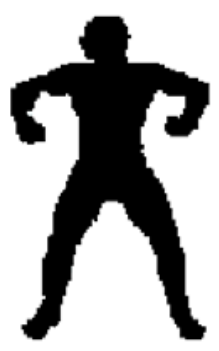

(a)

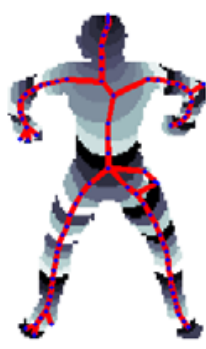

(b)

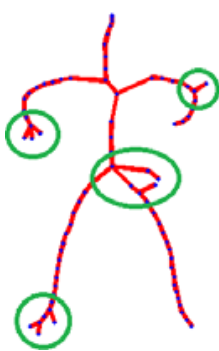

(c)

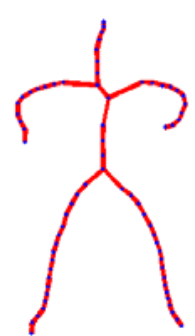

(d)

Fig. 3. Voxel scooping demonstrated on a $2 \mathrm{D}$ silhouette (in $3 \mathrm{D}$ the silhouette corresponds to object voxels): (a) Silhouette image. (b) Scooped levels and the resulting graph. (c) Graph with markings for some scooping errors. (d) pruned skeleton graph.

allows the user to rotate the camera around him- or herself by hand gestures. Finally, we use an image based visual hull (IBVH) technique to render the image of the user using the virtual camera. The remainder of this section describes important components of our system in more detail.

\subsection{D Reconstruction and Robust Skeleton Estimation}

In our system, we use a 3D model to determine the position of the head and hands of the user. We use a fast space carving approach on the GPU 13 to obtain a discrete voxel grid $V$ with a resolution of $k^{3}$ voxels $(k=64)$. Once the voxel model has been obtained, we need to perform a hierarchical segmentation into head, body and limbs. In the recent years it has become popular to build a so-called Reeb graph [19] to represent the topology of arbitrary multidimensional structures. A Reeb graph is generated by extracting level sets from such structures and keeping track of critical points where a level set becomes disconnected. When applied to the human body, an optimal Reeb graph has a tree structure with branches for head, arms and legs.

In this work, we utilize a fast and robust method called Voxel Scooping [12] for extracting the Reeb graph of the human body. While the algorithm was originally intended for tracing centerlines of neurons in medical data, we show that it can be easily applied to volumetric body scans such as obtained from our shape-from-silhouette approach. Starting with the node at a seeding point, each node in the graph spherically expands in voxel space with a locally adaptive radius (hence the name scooping). Based on this expansion, a new node is created and the process is repeated until there are no more voxels left to scoop. When the expanded voxels of a single node no longer form a connected component, a branching node is created and each branch is processed individually. In the final Reeb graph there will be artifacts in the form of short branches (see Fig. 3c). A final pruning step removes branches that only consist of two or less nodes in order to obtain a clean graph with only six arcs corresponding to head, body and limbs (as in Fig. 3d). We propose to use the top of the head as the seeding 


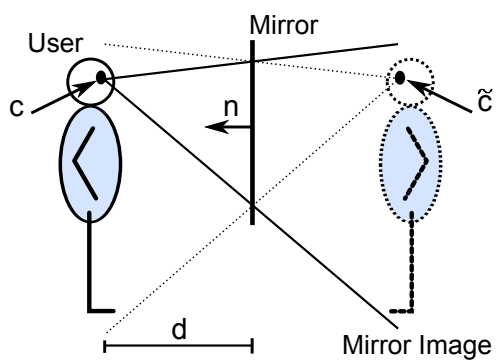

(a)

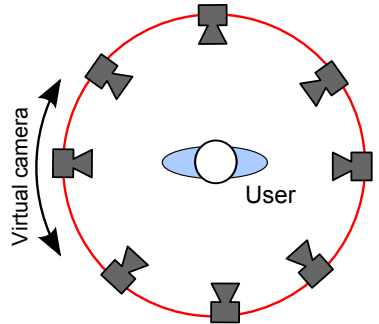

(b)

Fig. 4. (a) Illustration of the mirror effect with the position of the eyes $\mathbf{c}$, the position of the virtual camera $\tilde{\mathbf{c}}$ as well as the distance $d$ between the user and the mirror surface plus the mirror normal $\mathbf{n}$. (b) shows how the virtual camera is rotated around the user in free-viewpoint mode.

point for voxel scooping, which will always produce a tree structure similar to the human skeleton as in Fig. 3b. The position of the head is determined when the user steps into the system and is further tracked in voxel space.

\subsection{Hand Position Estimation}

For user interaction we use the position of the hands of the user, which we detect in the skeletal Reeb graph. The detection must work independently from the current body pose while being robust towards graph artifacts that cannot be removed using our length based graph pruning alone. Therefore, we propose to use the geodesic distance between leaf nodes as a pose-invariant feature to detect hands in the graph. Suppose there are $N_{E}$ leaf nodes in the graph, then $\left.p d(n)\right|_{n=1 \ldots N_{E}}$ is the shortest path-distance from node $n$ to the root of the graph (which is the head). In order to classify a leaf node as a hand, we compare each $p d(n)$ to the expected geodesic distance between the head and hands which is approximately 0.6 times the body height. During voxel scooping, geodesic distances between all nodes and the root are calculated automatically, therefore no further processing is required (such as building shock graphs). Note that the graph will not completely represent the full human body topology in the general case. For example, arms will not be distinguishable from the upper body in the voxel model when they are close to the body. However, for user-interaction we are only interested in hands that are stretched away from the body and therefore we can ignore hands in undetectable poses.

\subsection{Virtual Mirror Camera}

A mirror image as seen by the user depends on two factors: the position of the eyes $\mathbf{c} \in \mathbb{R}^{3}$ and the mirror plane $\pi \in \mathbb{R}^{4}$. While the mirror plane is fixed and configured once, the position of the eyes changes when users move. For an authentic mirror simulation, it is sufficient to approximate the position of the 
eyes by adding a constant offset to the top of the skeletal graph in every frame. The mirror plane $\pi$, which is the surface of the monitor in our system, can be expressed using a homogeneous notation which satisfies $\pi^{T} \mathbf{X}=0$ for every $3 \mathrm{D}$ point $\mathbf{X}$ that lies on the plane. The first three components of $\pi$ correspond to the plane normal

$$
\mathbf{n}=\left[\pi^{(1)}, \pi^{(2)}, \pi^{(3)}\right]^{T} \quad\|\mathbf{n}\|=1
$$

of the mirror while the fourth component $\pi^{(4)}$ describes the distance of the plane from the origin as determined in the configuration step. The orthogonal distance $d$ between $\mathbf{c}$ and the mirror surface can be calculated as follows:

$$
d=\pi^{T}\left[\begin{array}{l}
\mathbf{c} \\
1
\end{array}\right] .
$$

The $3 \times 4$ camera matrix $\tilde{\mathbf{P}}=\tilde{\mathbf{K}}[\tilde{\mathbf{R}} \mid \tilde{\mathbf{t}}]$ producing an optically correct mirror image from the user's view on the monitor can be calculated from the position of eyes $\mathbf{c}$, the distance $d$ and the normal $\mathbf{n}$ of the mirror plane (see Fig. 4a ). The rotation $\tilde{\mathbf{R}}_{[3 \times 3]}$ of the virtual camera is defined so that the camera looks along the normal $\mathbf{n}$ of the mirror surface with an up-vector $\left(\mathbf{u p}=[0,0,1]^{T}\right)$ :

$$
\tilde{\mathbf{R}}=\left[\begin{array}{c}
\mathbf{r}_{x}^{T} \\
\left(\mathbf{r}_{x} \times \mathbf{n}\right)^{T} \\
\mathbf{n}^{T}
\end{array}\right] \text { with } \quad \mathbf{r}_{x}=\mathbf{n} \times \mathbf{u p}
$$

Note that the gazing direction of the user does not affect the mirror image and therefore does not need to be considered. The position $\tilde{\mathbf{c}}$ of the virtual camera is simply a mirrored version of the position of the eyes $\mathbf{c}$ which yields the translation vector $\tilde{\mathbf{t}}$ for the virtual projection matrix $\tilde{\mathbf{P}}$ :

$$
\tilde{\mathbf{t}}=-\tilde{\mathbf{R}} \cdot \tilde{\mathbf{c}} \quad \text { with } \quad \tilde{\mathbf{c}}=\mathbf{c}-2 \cdot d \cdot \mathbf{n} .
$$

The last component of the camera matrix $\tilde{\mathbf{P}}$ is the intrinsic matrix $\tilde{\mathbf{K}}$ which mirrors and projects the scene onto the monitor's surface:

$$
\tilde{\mathbf{K}}=\left[\begin{array}{ccc}
-f & 0 & p_{x} \\
0 & -f & p_{y} \\
0 & 0 & 1
\end{array}\right] \quad \text { with } \quad f=2 \cdot d
$$

The focal length $f$ of the camera is equal to the distance between the virtual camera and the user, which is twice the user-mirror distance $d$. This can be explained by the observation that the size of oneself's image on the mirror surface is constant and independent from $d$ and the focal length of the eyes (see Fig. 1b). In the general case, the mirror image will be an off-center projection with the principal point offsets $p_{x}$ and $p_{y}$ shifted depending on the eye position c, the size of the monitor and its resolution in pixels as well as the position of the application window on the monitor. 


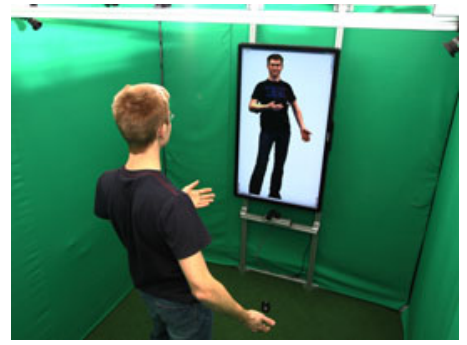

(a)

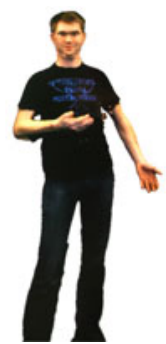

(b)

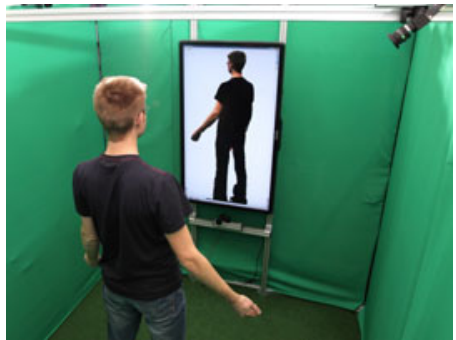

(c)

Fig. 5. (a) Simulation of a mirror with the generated mirror image in (b). (c) The user is looking at his own back.

\subsection{User Interaction}

Apart from the mirror simulation, our system supports a full $360^{\circ}$ view of the user by rotating the virtual mirror camera around the body as seen in Fig. 4b, We propose a simple and intuitive way of controlling this view through hand gestures. The user can trigger a clockwise or counterclockwise rotation of the mirror image by stretching his left or right hand away from his body, respectively. Stretching out both hands resets the rotation and sets the camera back to the normal mirror mode. Such input can be implemented by detecting hand positions as described in Sect. 4.2 and does not require visible markers attached to the human body. In order to minimize unintentional user inputs, we require the user to maintain a certain pose for a short amount of time (e.g. one second) before an action is triggered.

\subsection{Image-Based Visual Hull Rendering}

For mirror image rendering, our system uses an efficient implementation of the image-based visual hull (IBVH) algorithm by Matusik et al. [9] which only needs to calculate those parts of the visual hull that appear in the rendered image. Similar to [18, it is entirely implemented on the GPU but offers a set of additional features: our algorithm can process images from cameras that do not see the entire body of the user. Moreover, we implemented a view-dependent texture mapping scheme including a visibility check and stereo matching which allow us to improve the visual quality of the output. Implementation details about this rendering module can be found in 7 .

\section{Experiments}

In this section we evaluate our virtual mirror system in terms of visual quality, robustness and run-time. Figure 5 a shows an example of an user interacting with the system. As soon as the user steps in front of the monitor, a virtual mirror 


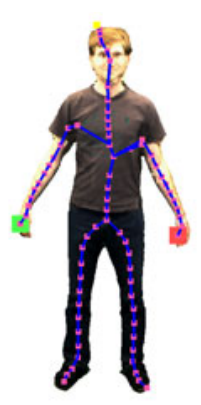

(a)

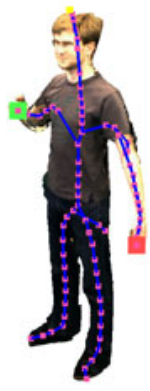

(b)

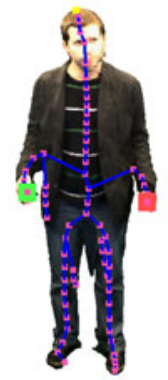

(c)

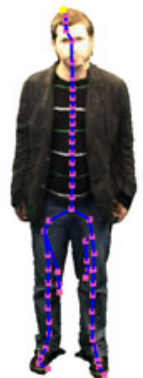

(d)

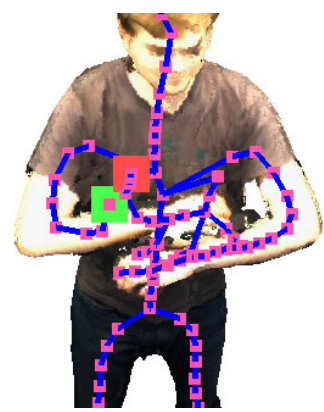

(e)

Fig. 6. Evaluation of skeleton extraction by voxel scooping and hand detection on different poses. (a) to (c) show correct skeleton and hand detection. In (d) the arms are merged with the body and no hands are detected. (e) shows wrong hand detections due to errors in the voxel model and skeleton.

image such as in Fig. 5 b is shown on the screen. We obtain an appealing artificial mirror image at a resolution of $540 \times 960$ pixels, from any virtual camera position such as in Fig. $5 \mathrm{c}$ which shows a user looking at his own back.

\subsection{User Interaction}

In our system, we implement marker-less user interaction through hand gestures. As we rely only on a very simplistic model of the human body (it must contain a head and arms) we can robustly detect the hands when they are represented correctly in the underlying skeletal graph. This is shown in Fig. 6 (a) to (c). When the arms are too close to the body, they cannot be distinctively detected in the skeletal graph anymore (Figure 6d). Similarly, when the arms have multiple connections to the body in voxel space (as in Fig. 6e), the skeleton reconstruction fails and wrong detections can occur. However, such errors will only appear when the hands are close to the body. As we react to user input only when hands are distinctly away from the body, we can ignore these errors.

\subsection{Run-Time Evaluation}

For an interactive system, fast processing and a low latency between user input (or movement) and graphical output is desirable. A balanced use of graphics hardware and CPU processing allows us to achieve real-time execution for the whole system. We are able to render an output mirror image at 15 frames per second and display it to the user less than $100 \mathrm{~ms}$ after camera image acquisition. A detailed evaluation of the average run-time of individual components can be found in Table 1 where the total runtime of all components is below $1 / 15 \mathrm{~s}$ or $66 \mathrm{~ms}$. In contrast to other systems such as [1]6], we require only a single computer for processing the camera images. Also, we do not need to process a 
Table 1. Run-time of the individual components averaged over 100 frames

\begin{tabular}{lcr}
\hline Component & Processor Runtime \\
\hline Undistortion and Segmentation & GPU & $6.1 \mathrm{~ms}$ \\
Voxel Space-carving $\left(64^{3}\right.$ voxels $)$ & GPU & $4.2 \mathrm{~ms}$ \\
Skeletal graph and hand detection & CPU & $5.7 \mathrm{~ms}$ \\
Rendering & GPU & $32.4 \mathrm{~ms}$ \\
\hline Total & & $48.4 \mathrm{~ms}$ \\
\hline
\end{tabular}

high resolution volumetric representation of the user as rendering of the output is performed on an image-based visual hull. For Reeb graph generation and hand detection, a $64^{3}$ voxel model is sufficient.

\section{Conclusions and Discussions}

We have presented a system which displays a convincingly and optically correct mirror image of the user who is standing in front of a large monitor. This has been achieved through a free-viewpoint video system and visual hull based rendering. In order to interact with the system, around 15 frames per second and low latencies are desirable which we are able to achieve on a single computer using simple image features (silhouettes) and high performance processors (GPU). The key benefit in using the GPU is the parallelization of image processing and rendering tasks, which account for more than 80 percent of our computing workload. Besides the simulation of a real mirror, we allow the user to look at him- or herself from an arbitrary viewpoint and control this behavior through hand gestures. We realize this through a skeleton like representation of the user in which we detect the hands robustly without the need for visual markers.

To our knowledge, our system is the first virtual mirror that accurately simulates a real mirror. The best existing mirror simulation so far is [6] where the facial image of a single fixed camera is transformed and displayed by assuming the face lies on a plane parallel to the monitor. Our system does not require any transformations in image space, which facilitates integration of artificial objects that do not lie on the facial plane but can be placed anywhere in space. While all existing virtual mirror systems allow real-time operation by processing $2 \mathrm{D}$ images, we produce a similar output but from a full 3D representation of the user within the same time constraints. Unlike marker based systems such as [5], users do not have to wear or carry objects in order to interact with our system. We allow user interaction in 3D space using no more than their hands.

The current implementation is limited to displaying only the user as seen by the cameras from an arbitrary viewpoint. In future work we will integrate artificial objects such as virtual clothes into the mirror image and let users interact with them. Also, our multi-view camera system allows for generation of high quality $3 \mathrm{D}$ scans and motion capture. 
Acknowledgements. This work was supported by the Austrian Research Promotion Agency (FFG) under the BRIDGE program, project \#822702.

\section{References}

1. Allard, J., Franco, J.S., Ménier, C., Boyer, E., Raffin, B.: The GrImage Platform: A Mixed Reality Environment for Interactions. In: Proc. of the International Conference on Computer Vision Systems (2006)

2. Darrell, T., Gordon, G., Woodfill, J., Harville, M.: A virtual mirror interface using real-time robust face tracking. In: Proc. of the International Conference on Automatic Face and Gesture Recognition (1998)

3. De Aguiar, E., Stoll, C., Theobalt, C., Ahmed, N., Seidel, H.P., Thrun, S.: Performance capture from sparse multi-view video. In: Proc. of ACM SIGGRAPH (2008)

4. Eisert, P., Rurainsky, J., Fechteler, P.: Virtual mirror: Real-time tracking of shoes in augmented reality environments. In: Proc. of the ICIP (2007)

5. Fiala, M.: Magic mirror system with hand-held and wearable augmentations. In: Proc. of the IEEE Virtual Reality Conference, pp. 251-254 (2007)

6. François, A.R., Kang, E.: A handheld mirror simulation. In: Proc. of the International Conference on Multimedia and Expo., vol. 2, pp. 745-748 (2003)

7. Hauswiesner, S., Straka, M., Reitmayr, G.: Coherent image-based rendering of real-world objects. In: Proc. of the Symposium on Interactive 3D Graphics (2011)

8. Hilsmann, A., Eisert, P.: Realistic cloth augmentation in single view video. In: Proc. of Vison, Modelling, and Visualization Workshop, pp. 55-62 (2009)

9. Matusik, W., Buehler, C., Raskar, R., Gortler, S., McMillan, L.: Image-based visual hulls. In: Proc. of ACM SIGGRAPH, pp. 369-374 (2000)

10. Nickolls, J., Dally, W.: The GPU computing era. IEEE Micro 30(2), 56-69 (2010), doi:10.1109/MM.2010.41

11. Ray-Ban Sunglasses: Virtual mirror, from http://www.ray-ban.com/usa/science/virtual-mirror (retrieved March 14, 2011)

12. Rodriguez, A., Ehlenberger, D., Hof, P., Wearne, S.L.: Three-dimensional neuron tracing by voxel scooping. Journal of Neuroscience Methods 184(1), 169-175 (2009), doi:10.1016/j.jneumeth.2009.07.021

13. Schick, A., Stiefelhagen, R.: Real-time GPU-based voxel carving with systematic occlusion handling. In: Denzler, J., Notni, G., Süße, H. (eds.) Pattern Recognition. LNCS, vol. 5748, pp. 372-381. Springer, Heidelberg (2009)

14. Starck, J., Hilton, A.: Surface capture for performance based animation. IEEE Computer Graphics and Applications 27(3), 21-31 (2007)

15. Tilley, A.R., Dreyfuss, H.: The Measure of Man \& Woman. John Wiley \& Sons, Chichester (2002)

16. Tzevanidis, K., Zabulis, X., Sarmis, T., Koutlemanis, P., Kyriazis, N., Argyros, A.: From multiple views to textured 3D meshes: a GPU-powered approach. In: Proc. of the Computer Vision on GPUs Workshop, CVGPU (2010)

17. del Valle, A.C.A., Opalach, A.: Enhanced reflection to encourage healthy living. In: Proc. of UbiComp Workshop: Monitoring, Measuring, and Motivating (2005)

18. Waizenegger, W., Feldmann, I., Eisert, P., Kauff, P.: Parallel high resolution realtime visual hull on GPU. In: Proc. of ICIP (2009)

19. Werghi, N., Xiao, Y., Siebert, J.P.: A functional-based segmentation of human body scans in arbitrary postures. IEEE Transactions on Systems, Man and Cybernetic 26(1), 153-165 (2006) 